\title{
Immunothérapie et syndrome oral
}

\section{Immunotherapy and oral syndrome}

\author{
D. Sabouraud-Leclerc* ${ }^{1}, \mathrm{X}$. Van der Brempt ${ }^{2}$ \\ ${ }^{1}$ CHU de Reims, service de pédiatrie générale et spécialisée, \\ 47 rue Cognacq-Jay, 51100 Reims, France \\ ${ }^{2}$ Allergopôle, Clinique Saint-Luc, Bouge (Namur), Belgique
}




\title{
Résumé
}

L'allergologue rencontre dans sa pratique de plus en plus de syndromes oraux (SO) aux fruits (Rosacées) et légumes crus, suite à l'augmentation des pollinoses (Bétulacées). Si ces SO se limitent en général à une gêne buccale, ils altèrent notablement la qualité de vie des patients atteints et posent le problème de leur prise en charge : plutôt que l'éviction, n'est-il pas possible de mettre en place une immunothérapie orale et comment?

Mots-clés : Immunothérapie; syndrome oral; Bétulacées; pomme

\begin{abstract}
In their daily practice, allergists are increasingly seeing oral syndrome (OS) involving fruit (Rosaceae family) and raw vegetables following an increase in pollinosis (Betulaceae family). Although oral syndromes are generally limited to oral discomfort, they in fact cause notable impairment of the quality of life of patients presenting the syndrome and pose the problem of therapy: rather than withholding the offending foodstuffs, would it not be possible to institute oral immunotherapy, and if so, how?
\end{abstract}

Keywords: immunotherapy; oral syndrome; Betulaceae; Apple

Le syndrome oral (SO) ou «Pollen Food Syndrome » est dû à une réaction croisée entre des protéines alimentaires d'origine végétale et des pollens. Ces réactions croisées sont liées à de fortes homologie de séquence et/ou structurales d'où des épitopes communs.

Ces allergènes sont des panallergènes retrouvés en abondance dans le règne végétal, tels les PR10, pathogenesis-related proteins, responsables de la majeure partie des SO via les pollens de bétulacées et, moins fréquemment les profilines, présentes dans le pollen des bétulacées, mais aussi des graminées et de l'ambroisie [1].

Cliniquement, le SO se manifeste par un prurit de la sphère buccale, un oedème des lèvres voire un prurit pharyngé survenant dans les minutes suivant la consommation de l'aliment: pomme, pêche, cerise, mais aussi noisette, arachide, plus rarement noix (profiline), épices,... d'où un arrêt instinctif de consommation et des symptômes limités à la sphère buccale [2]. Les protéines PR10 sont détruites par la chaleur (et l'acidité gastrique), ce qui explique que la plupart des patients tolèrent les aliments cuits : compotes, pâte à tartiner de noisette. . . Cependant, des réactions plus sévères sont décrites avec des légumes (céleri, carottes, soja) riches en PR10 ou en présence de cofacteurs [3].

Si les premiers cas ont été décrits chez l'adulte, les syndromes oraux sont de plus en plus fréquents chez l'enfant via l'augmentation des pollinoses liées aux modifications climatiques (rôle de la pollution notamment) [2]. 
Ces réactions sont donc le plus souvent bénignes, se limitant à un « désagrément » buccal mais elles altèrent notablement la qualité de vie du patient, l'empêchant de consommer de nombreux fruits voire légumes, riches sur le plan nutritionnel et agréables au goût.

Les allergologues peuvent-ils agir sur le syndrome oral ? Dans cette présentation, nous nous limiterons à l'immunothérapie en cas de syndrome oral lié aux pollens de Bétulacées, cas de loin le plus fréquent et ayant fait l'objet de publications.

La revue de la littérature montre que l'immunothérapie spécifique aux pollens de bétulacées mise en place pour traiter la pollinose n'a finalement que très peu d'impact sur le SO.

Cependant, pour certains, cette immunothérapie spécifique a pu améliorer le syndrome oral [4], pour d'autres, elle l'aggrave ou n'a pas d'effet [5]. L'étude randomisée récente de Kinaciyan et coll, portant sur 60 patients adultes allergiques aux pollens de bétulacées présentant un syndrome oral à la pomme et mis en immunothérapie sublinguale (ITSL) soit par recombinant de la pomme $\mathrm{r}$ Mal d 1, soit par le recombinant du bouleau r Bet v1, ou par placebo montre que seule l'ITSL avec r Mal d1 diminue les syndromes oraux alors qu'il n'y a pas d'efficacité avec r Bet v1 ni avec le placébo. Cependant l'efficacité est partielle puisque seuls 5/20 (25\%) patients du groupe r Mal d 1 sont capables de tolérer 20g de pomme, 14 (70\%) ont amélioré leur tolérance, 4 n'ont pas progressé et 2 ont diminué leur tolérance à la pomme [6].

D'autres équipes ont travaillé sur des protocoles d'immunothérapie orale (ITO) à la pomme initiés soit à domicile après un TPO à la pomme, soit en rush de quelques heures sous surveillance médicale avec poursuite à domicile $[7,8]$ avec des résultats très intéressants. Bouvier et coll. ont ainsi proposé une ITO à la pomme à 52 patients adultes et enfants allergiques au bouleau, avec SO aux Rosacées responsable le plus souvent d'une éviction totale de ces fruits crus depuis plusieurs années. Après 24 semaines de progression des doses, 47 patients consommaient une demi-pomme, et après 48 semaines, 41 patients consommaient la pomme et la plupart des autres Rosacées de façon normale [7]. Van der Brempt et coll. ont utilisé un protocole accéléré sur une série de 132 patients; après 2 mois de progression de doses, 128/132 patients ont pu consommer une pomme Golden entière, et moyennant la poursuite d'une consommation régulière de pommes, la plupart ont maintenu leur tolérance orale aux Rosacées avec un suivi allant jusqu'à 4,5 ans [8].

La plupart des auteurs s'accordent cependant pour dire que cette ITO à la pomme ne permet pas d'obtenir une véritable désensibilisation, mais seulement une tolérance, et que cette tolérance ne se maintient que si la consommation des rosacées reste suffisamment fréquente et importante.

\section{Références :}

[1] Pauli G. Allergènes végétaux alimentaires identifiés (en dehors de l'arachide). Rev Fr Allergol 2011;51:56-62.

[2] Werfel T, Asero R, Ballmer-Weber BK et al. Position paper of the EAACI: food allergy due to immunological cross-reactions with common inhalant allergens. Allergy 2015; 70:1079-90.

[3] Sabouraud-Leclerc D, Bradatan E. Des PR 10 pas si anodines, à propos de quelques cas pédiatriques. Rev Fr Allergol 2017;57:437-41. 
[4] Mauro M, Russello M, Incorvaia C, Gazzola G, Frati F, Moingeon P, et al. Birch-apple syndrome treated with birch pollen immunotherapy. Int Arch Allergy Immunol 2011;156:41622.

[5] Kinaciyan T, Jahn-Schmid B, Radakovics A, Zwölfer B, Schreiber C, Francis JN, et al. Successful sublingual immunotherapy with birch pollen has limited effects on concomitant food allergy to apple and the immune response to the Bet v 1 homolog Mal d 1. J Allergy Clin Immunol 2007;119:937-43.

[6] Kinaciyan T, Nagl B, Faustmann S, Frommlet F, Kopp S, Wolkersdorfer M, Wöhrl S et al. Efficacy and safety of 4 months of sublingual immunotherapy with recombinant Mal d 1 and Bet v 1 in patients with birch pollen-related apple allergy. J Allergy Clin Immunol 2018;141:1002-8.

[7] Bouvier M, Van der Brempt X, Nosbaum A, Cordier J-M, Cherih C, Frappaz A, et al. L'induction de tolérance orale dans l'allergie aux rosacées. Rev Fr Allergol 2014;54:127-33. [8] Van der Brempt X, Bradatan E, Vandezande LM, Frognier R. Induction de tolérance orale (ITO) à la pomme: tolérance et efficacité à long terme sur le syndrome oral chez 132 patients. Rev Fr Allergol 2018;58:288. 